\title{
McRAPD unlike MALDI-TOF MS is a suitable candidate for routine discrimination of new Haemophilus influenzae strain acquisition in chronic obstructive pulmonary disease (COPD) and cystic fibrosis
}

\author{
Vladislav Raclavskya,b, Nikola Stromerovac, Dana Safarovad, Jan Bardonc, Jaromir Zatloukale , Martin Zapalka , Petr Jakubece, \\ Lucie Navratilova ${ }^{a}$, Radko Novotny ${ }^{a}$
}

\begin{abstract}
Background and Aims. Haemophilus influenzae new strain acquisition has been demonstrated to increase the relative risk of acute exacerbation fourfold in contrast to colonisation or chronic infection by the same strain in chronic obstructive pulmonary disease (COPD). Unfortunately, molecular typing techniques are not suitable for routine use due to cost, labour-intensity and need for special expertise. We tested two techniques potentially useful for routine typing, namely the newly available MALDI-TOF MS and the modified MCRAPD compared to MLST as the gold standard. Methods. In 10 patients (10.8\%) suffering from COPD or cystic fibrosis, $H$. influenzae isolates were recovered repeatedly at different timepoints from the same patient during the study period. This allowed for thirteen pairwise comparisons of typing results in isolates recovered consecutively from the same patient to test the ability of the techniques to uncover new strain acquisition.

Results. MLST detected 9 cases of new strain acquisition among the 13 pairwise comparisons. However, MALDI-TOF MS reported all 13 pairs as different and thus new. In contrast, McRAPD was able to differentiate all the new strain acquisitions from pre-existing ones, both by visual inspection of melting profiles and by Relative Significant Difference values. Conclusions. Unlike MALDI-TOF MS, MCRAPD appears to be a suitable candidate for routine discrimination of new strain acquisitions because of its accuracy and, rapid, easy and economic performance.
\end{abstract}

Key words: Haemophilus influenzae, chronic obstructive pulmonary disease, cystic fibrosis, MALDI-TOF MS typing, McRAPD typing, high resolution melting analysis

Received: January 22, 2016; Accepted with revision: September 21, 2016; Available online: October 6, 2016

http://dx.doi.org/10.5507/bp.2016.050

${ }^{a}$ Department of Microbiology, Faculty of Medicine and Dentistry, Palacky University Olomouc, Czech Republic

bInstitute of Molecular and Translational Medicine, Faculty of Medicine and Dentistry, Palacky University Olomouc, Czech Republic

'State Veterinary Institute Olomouc, Czech Republic

${ }^{d}$ Department of Cell Biology and Genetics, Faculty of Science, Palacky University Olomouc, Czech Republic

${ }^{e}$ Department of Respiratory Medicine, University Hospital Olomouc, Czech Republic

${ }^{f}$ Department of Pediatrics, University Hospital Olomouc, Czech Republic

Corresponding author: Vladislav Raclavsky, e-mail:vladislav.raclavsky@upol.cz

\section{INTRODUCTION}

Haemophilus influenzae represents an important pathogen often found in the respiratory pathways of COPD patients. However, the interpretation of such a finding remains difficult because it is frequently present both in stable disease and during exacerbations. In such cases, new bacterial strain acquisition has been suggested as a possible cause of exacerbations. Significant association of new $H$. influenzae strain acquisition with exacerbation ( $26.2 \%$ of visits) versus no new strain ( $17.1 \%$ of visits, $P<$ 0.001 ) was first documented by Sethi et al. ${ }^{1}$. Furthermore, the newly acquired strains have been documented to elicit development of strain-specific serum antibodies, whereas same strains were not associated with such response in the majority of instances ${ }^{2}$. Later, the same group recognized that some isolates of $H$. influenzae were actually $H$. haemolyticus species. When the results of the study were reanalysed, there was no association of new H. haemolyticus acquisition and exacerbation ( $20.7 \%$ vs. $17.4 \%, P=0.47)$, whereas the association between the acquisition of a new strain of $H$. influenzae and acute exacerbations increased to $44.5 \%$ vs. $16.4 \%(P<0.001)\left(\right.$ ref. $\left.^{3}\right)$. These findings should allow for better targeting of therapy and provide the possibility to improve clinical outcomes and modify disease activity in COPD (ref. $\left.{ }^{4}\right)$. Unfortunately, discrimination of new versus same strains relies on molecular typing techniques that are typically costly, labour-intensive, and need special expertise. Therefore, their use is at present limited to research studies. However, with the advent of new technologies, namely whole bacterial cell Matrix-assisted laser desorption/ionisation-time of flight mass spectrometry (MALDI-TOF MS) and Melting curve analysis of RAPD (McRAPD), diagnostic laboratories might be able to perform strain typing routinely in the foreseeable future. The aim of our work was to explore the potential of MALDI-TOF MS protein profiling and McRAPD (ref. ${ }^{5}$ ) in discrimination between the newly acquired and pre-existing $H$. influenzae isolates recovered from sputum of patients suffering from COPD or cystic fibrosis (CF). 


\section{MATERIAL AND METHODS}

\section{Study design}

The Ethics Committee of the University Hospital Olomouc approved the study protocol. All participants gave written informed consent. Sputum samples or nasal and pharyngeal swabs were collected from COPD and CF patients who sought medical care at visits or during hospital stays at the Dept. of Respiratory Medicine and Dept. of Pediatrics, University Hospital Olomouc during the period from April 2012 to October 2014.

\section{Cultivation and species identification}

Sputum samples were liquefied by adding a roughly equal volume of $2 \% \mathrm{~N}$-acetylcysteine solution (typically 5-10 $\mathrm{mL}$ ) and homogenized by shaking with glass beads, diluted and inoculated on Haemophilus selective agar (Oxoid). For economic reasons, 104-times dilution was plated on one half of the plate and $10^{8}$-times dilution was plated on the other half of the same plate to facilitate differentiation of colonies at higher loads. Nasal and pharyngeal swabs were inoculated on Haemophilus selective agar (Oxoid) as well. All colonial morphotypes were identified using a MALDI-TOF MS Biotyper (Bruker, Germany) in accordance with manufacturer's instructions. All $\mathrm{H}$. influenzae isolates were subcultured and stored at $-70{ }^{\circ} \mathrm{C}$. The $H$. influenzae isolates cultured from the same patients at different timepoints during the study period were included into a further analysis as described below.

\section{Conventional typing}

Conventional serotyping was performed using the Haemophilus influenzae Antisera Set (Denka Seiken, Japan) in accordance with manufacturer's instructions.

\section{MLST typing}

Multilocus sequence typing (MLST) was performed as a gold standard typing technique according to the scheme developed by Meats et al. ${ }^{6}$ and with the use of the MLST database developed by Jolley and Maiden ${ }^{7}$. For economic reasons, the analysis was started with sequencing of the most variable locus $m d h$ ( 36 alleles), followed by the second most variable locus $p g i$ ( 32 alleles), etc. In most cases, sequencing of all loci was not necessary to detect new strain acquisition.

\section{MALDI-TOF MS TYPING}

Each isolate of $H$. influenzae was cultured as three replicates on Chocolate agar with Vitox (Oxoid) and subsequently processed into protein extracts by the Bruker's MALDI Biotyper Standard Operating Procedure: Ethanol and Formic Acid Extraction Method. Each protein extract was used to create the Main Spectrum (MSP) as follows: it was placed on 8 spots of a stainless steel target plate, overlaid with HCCA matrix ( $\alpha$-cyano-4hydroxycinnamic acid, Sigma-Aldrich) and measured as $3 \times 240$ shots per spot, i.e. 5760 shots per extract, using a
Microflex LT system (Bruker, Germany). Those spectra with outlier peaks or anomalies were removed from the set. Then, peaks from 3000 to $10,000 \mathrm{Da}$ with maximum mass error of $500 \mathrm{ppm}$ were selected. Software settings for MSP and dendrogram creation were those suggested by the Bruker's MALDI Biotyper 3.1 software (BioTyper Standard Method). The hierarchical cluster analysis and dendrogram construction was performed using default correlation function within the MALDI Biotyper 3.1 software package. To discriminate between continued presence of the same strain and acquisition of a new strain, a pairwise comparison was performed between $H$. influenzae isolates recovered from the same patient at two consecutive timepoints. Briefly, three MSPs generated from the three replicates of the protein extracts from one isolate and the same from the other isolate were together subjected to hierarchical cluster analysis and a dendrogram was constructed then. If the replicates of one isolate and those of the other isolate clustered separately, the two isolates were considered to be different, which indicated the acquisition of a new strain. If no such clustering, as mentioned before, occurred, the two isolates were considered to belong to the same strain. Because it was not possible to process all isolates in one run, three isolates were always processed per run, which included one pair of two consecutive isolates and one other unrelated isolate for comparison.

\section{McRAPD typing}

DNA was extracted using the GeneProof PathogenFree DNA PCR Kit (GeneProof, Czech Republic) in accordance with the manufacturer's instructions. One microliter of DNA was added into the amplification reaction that was performed in a $20 \mu \mathrm{L}$ volume using the qPCR $2 \times$ SYTO-9 Master Mix (Top-Bio, Czech Republic) with the $\mathrm{MgCl}_{2}$ concentration increased to $3.75 \mathrm{mmol} / \mathrm{L}$ and using $2.5 \mu \mathrm{mol} / \mathrm{L}$ of the 10 -nucleotide RAPD primer AP-12 (5'-CGGCCCCTGC-3'), which had been used as a RAPD primer for $H$. influenzae previously ${ }^{8}$. After incubation at $94{ }^{\circ} \mathrm{C}$ for $5 \mathrm{~min}$, the reaction mixtures were cycled 40 times through the following temperature profile: $95^{\circ} \mathrm{C}$ for $30 \mathrm{~s}, 43^{\circ} \mathrm{C}$ for $30 \mathrm{~s}$, and $72{ }^{\circ} \mathrm{C}$ for $60 \mathrm{~s}$. A LightCycler 96 (Roche) was used for amplification; slow ramping from the annealing to the extension temperature $\left(4^{\circ} \mathrm{C} / 1 \mathrm{~min}\right.$, i.e. $7 \mathrm{~min}$ for transition from 43 to $72{ }^{\circ} \mathrm{C}$ ) was used to enhance the reproducibility of RAPD as described by Benter et al. ${ }^{9}$. High resolution melting analysis (HRMA) was performed using the same instrument with initial denaturation at $95^{\circ} \mathrm{C}$ for $60 \mathrm{~s}$, renaturation at $40^{\circ} \mathrm{C}$ for 60 ${ }^{\circ} \mathrm{C}$ and heating to $70{ }^{\circ} \mathrm{C}$, followed by HRMA in the range $70-90{ }^{\circ} \mathrm{C}$ at highest ramp rate $\left(0.04{ }^{\circ} \mathrm{C} / \mathrm{s}\right)$ and highest resolution (25 readings per second). The resulting melting curves were proportionally normalized in accordance with manufacturer's instructions with empirical adjustment of pre-melting range to $77-78^{\circ} \mathrm{C}$, post-melting range to 87.8 $88.5^{\circ} \mathrm{C}$ and manual temperature shift at 0.05 threshold. Complex curves of normalized melting peaks $(-\mathrm{dF} / \mathrm{dT}$ derivation of normalized melting data), consisting of 253 temperature points (HRMA fluorescence readings), were 
imported into spreadsheet and evaluated as described below. Each $H$. influenzae isolate (DNA sample) was processed in six parallel reactions (replicates) to be able to check for McRAPD reproducibility and to evaluate statistical significance of differences between complex curves of normalized melting peaks.

\section{Numerical processing of McRAPD data and statistical analysis}

Differences between complex curves of normalized melting peaks obtained with each pair of $H$. influenzae isolates recovered from the same patient at different timepoints were evaluated as follows: at each temperature point of HRMA, 6 relative fluorescence intensity values obtained with 6 replicates of one isolate were compared to 6 relative fluorescence intensity values obtained with 6 replicates of the other isolate using twosided t-test. Then, a $P$-value marking the significance of the difference between the two isolates was obtained at each temperature point, resulting in $253 P$-values for each comparison of complex melting curves. To evaluate the aggregate significant difference between the complex melting peak curves of the two isolates, relative percentage of $P$-values lower than $0.01(P<0.01)$ was calculated for each pair of isolates under comparison. This relative percentage represents the relative part of the curves that are significantly different and, for our purposes, we termed it "Relative Significant Difference" (RSD). See Table 1 in supplementary data for complete data including their numerical processing and statistical analysis. To test for the influence of the number of McRAPD replicates on the robustness of such comparison, RSD values were also calculated in silico to model the case when less than 6 parallel McRAPD reactions would be performed per isolate.

\section{RESULTS}

In 10 patients $(10.8 \%), H$. influenzae isolates were recovered repeatedly at different timepoints from the same patient, yielding 24 isolates during the study period. Unfortunately, in one case we were unable to revive one isolate after storage, then, one pair of isolates had to be excluded from the study. See Table 2 for summary of 22 repeatedly recovered isolates included into the study.

Typing results were compared in pairs of isolates recovered consecutively in each of the patients at different timepoints. For example, if 3 isolates were recovered from patient No. 2 at different timepoints (isolate 2-1 followed by $2-2$ followed by $2-3$ ), then isolate $2-2$ was compared to isolate 2-1 and isolate 2-3 was compared to isolate 2-2 to possibly reveal interstrain differences that would prove the acquisition of a new strain at each consecutive timepoint of recovery. Conventional serotyping was able to detect two cases of new strain acquisition and generally was of little use, because $52 \%$ of the isolates were nontypeable. Sequencing of the most variable $m d h$ locus enabled a reliable differentiation of new strain acquisition in 7 of the 13

Table 2. Summary of repeatedly recovered isolates included into the study and results of MLST and McRAPD.

\begin{tabular}{|c|c|c|c|c|c|c|c|c|c|}
\hline Patient & Diagnosis & $\begin{array}{l}\text { Date of } \\
\text { recovery }\end{array}$ & $\begin{array}{c}\text { Isolate } \\
\text { No. }\end{array}$ & $\begin{array}{c}m d h \\
\text { allele }\end{array}$ & $\begin{array}{c}p g i \\
\text { allele }\end{array}$ & ST & $\begin{array}{c}\text { Isolate } \\
\text { comparison }\end{array}$ & $\begin{array}{c}\text { MLST } \\
\text { conclusion }\end{array}$ & $\begin{array}{c}\text { McRAPD based } \\
\text { RSD (\%) }\end{array}$ \\
\hline \multirow[t]{4}{*}{ Patient 1} & $\mathrm{CF}$ & 6.6 .2013 & $1-1$ & 1 & - & - & & & \\
\hline & & 4.3.2014 & $1-2$ & 22 & 19 & 124 & $1-2$ versus $1-1$ & Different & 59.3 \\
\hline & & 25.3 .2014 & $1-3$ & 22 & 19 & 124 & $1-3$ versus $1-2$ & Identical & 16.5 \\
\hline & & 14.9.2014 & $1-4$ & 22 & 19 & 124 & $1-4$ versus $1-3$ & Identical & 0 \\
\hline \multirow[t]{4}{*}{ Patient 2} & $\mathrm{CF}$ & 10.5 .2013 & $2-1$ & 1 & - & - & & & \\
\hline & & 25.10 .2013 & $2-2$ & 7 & - & - & $2-2$ versus $2-1$ & Different & 73.4 \\
\hline & & 20.2 .2014 & $2-3$ & 22 & - & - & $2-3$ versus $2-2$ & Different & 85.9 \\
\hline & & 31.5 .2013 & $3-1$ & 26 & - & - & & & \\
\hline \multirow[t]{2}{*}{ Patient 3} & $\mathrm{CF}$ & 12.5 .2014 & $3-2$ & 88 & 63 & - & $3-2$ versus $3-1$ & Different & 49.8 \\
\hline & & 18.8.2014 & $3-3$ & 88 & 78 & - & $3-3$ versus $3-2$ & Different & 55.9 \\
\hline \multirow[t]{2}{*}{ Patient 4} & $\mathrm{CF}$ & 6.5 .2013 & $4-1$ & 26 & 61 & 396 & & & \\
\hline & & 3.3.2014 & $4-2$ & 26 & 61 & 396 & $4-2$ versus $4-1$ & Identical & 17.5 \\
\hline \multirow[t]{2}{*}{ Patient 5} & $\mathrm{CF}$ & 15.2 .2013 & $5-1$ & 81 & - & - & & & \\
\hline & & 29.3.2013 & $5-2$ & 81 & - & - & $5-2$ versus $5-1$ & Different & 79.1 \\
\hline \multirow[t]{2}{*}{ Patient 6} & $\mathrm{CF}$ & 27.5.2014 & $6-1$ & 9 & - & - & & & \\
\hline & & 30.6 .2014 & $6-2$ & 158 & - & - & $6-2$ versus $6-1$ & Different & 52.9 \\
\hline \multirow[t]{2}{*}{ Patient 7} & $\mathrm{CF}$ & 17.3.2014 & $7-1$ & 17 & - & - & & & \\
\hline & & 18.8 .2014 & $7-2$ & 7 & - & - & $7-2$ versus $7-1$ & Different & 77.4 \\
\hline \multirow[t]{2}{*}{ Patient 8} & COPD & 25.6 .2013 & $8-1$ & 9 & 14 & 11 & & & \\
\hline & & 13.11.2013 & $8-2$ & 9 & 14 & 11 & $8-2$ versus $8-1$ & Identical & 1 \\
\hline \multirow[t]{2}{*}{ Patient 9} & COPD & 25.10 .2012 & $9-1$ & 1 & 1 & - & & & \\
\hline & & 14.11 .2013 & $9-2$ & 1 & 21 & - & $9-2$ versus 9-1 & Different & 54.1 \\
\hline
\end{tabular}

$\mathrm{ST}=$ sequence type according to the MLST scheme 
pairwise comparisons. Further sequencing of more MLST loci in the remaining 6 pairs revealed only two other newly acquired strains, whereas strain identity was confirmed in the remaining 5 cases using complete MLST typing. See Table 2 for a summary of MLST results.
The results of MALDI-TOF MS typing represented by pairwise comparisons of MSPs are summarised in Fig. 1. Triplicates of MSPs generated from three independent protein extractions of one isolate and those generated from the other isolate were subjected to hierarchical cluster anal-

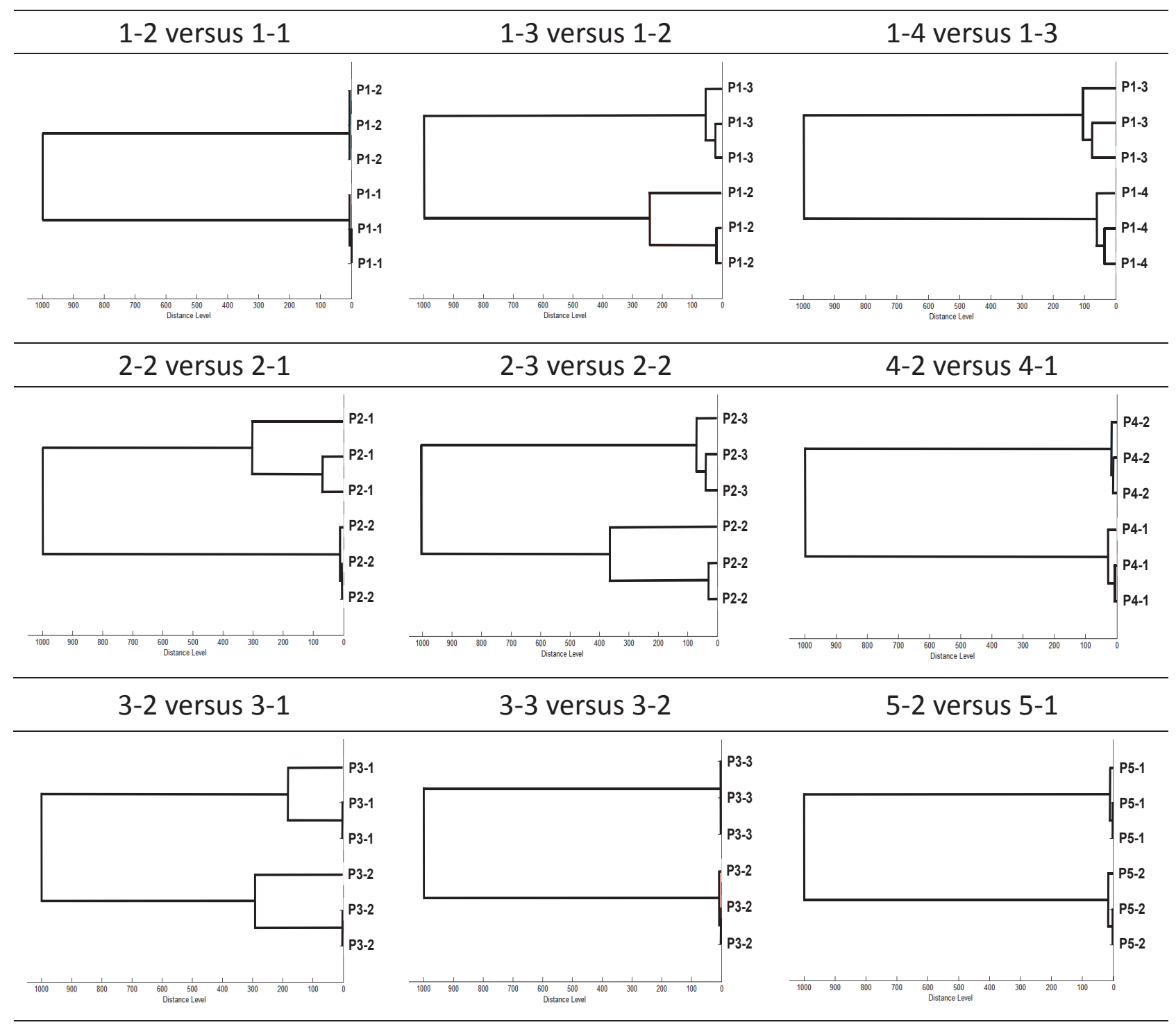

$6-2$ versus $6-1$

$7-2$ versus $7-1$

8-2 versus $8-1$

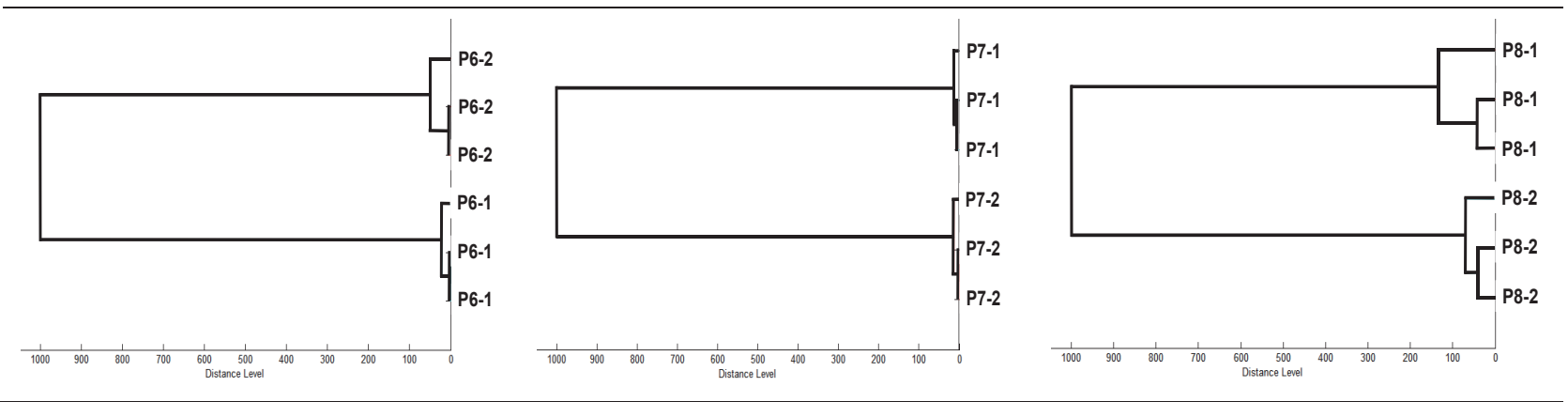

Fig. 1. Results of MALDI-TOF MS typing represented by pairwise comparisons of MSPs. Triplicates of MSPs generated from three independent protein extractions of one isolate and those generated from the other isolate were subjected to hierarchical cluster analysis and a dendrogram was constructed. Each dendrogram represents one pairwise comparison of MSPs, e.g. 1-2 versus 1-1 dendrogram represents comparison MSPs obtained with the isolate No. 2 from patient No. 1 versus isolate No. 1 from the same patient. Because of space limitations, twelve pairwise comparisons are demonstrated instead of all thirteen ones. 


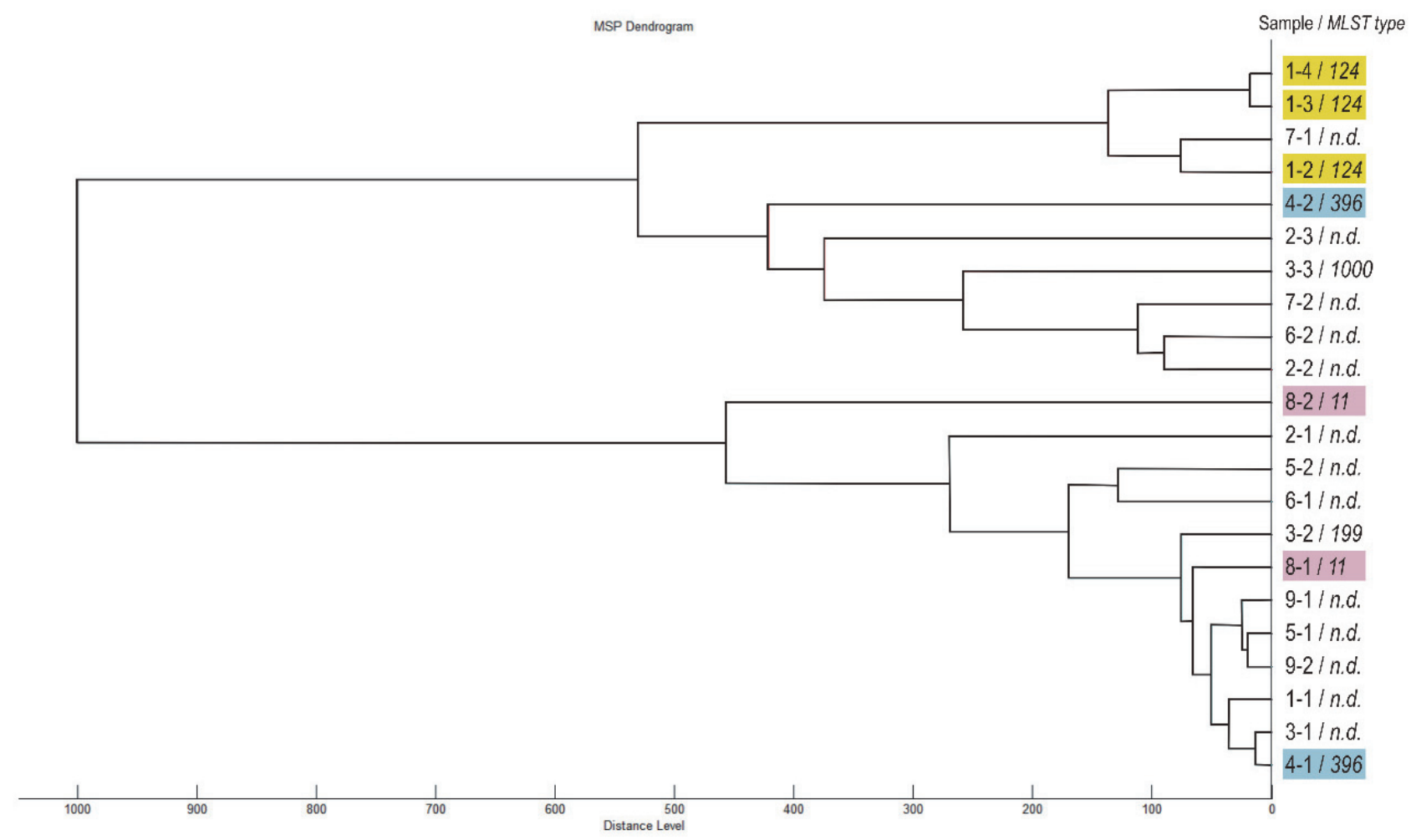

Fig. 2. Results of MALDI-TOF MS typing represented by complete dendrogram. Three main spectra (MSPs) were created from three replicate cultures of each isolate. These three spectra were merged and used for hierarchical cluster analysis and dendrogram construction using default correlation function within the MALDI Biotyper 3.1 software package. For reference, MLST types are listed in italics after slash; isolates that belong to the same MLST type are highlighted by the same colour.

ysis and a dendrogram was constructed. In all cases, the extracts of each particular isolate clustered together and were clearly separated from the cluster formed by the other isolate, indicating the acquisition of a new strain. In addition, complete dendrogram based on merged data from triplicates is demonstrated in Fig. 2. Isolates that belong to the same MLST type are highlighted by the same colour.

The results of McRAPD typing represented by complex melting peak curves are demonstrated in Fig. 3. Each plot represents a comparison of six replicates from one isolate versus six replicates from the other isolate, both from the same patient. Replicates obtained with the same isolate are coded by the same colour. Relative Significant Difference (RSD) values calculated from six versus six replicates are summarised in Table 2. The average RSD value reached $66.7 \pm 13.78 \%$ in pairs of isolates that were demonstrated to be different by MLST, which was clearly significantly different from the same value for pairs of isolates that belonged to the same strain according to MLST, namely $8.8 \pm 9.54 \%(P<0.001)$. RSD values calculated in silico, to model the situation when less than six replicates per isolate would be included, are summarised in Fig. 4.

\section{DISCUSSION}

Sequencing of just one MLST locus, namely the most variable $m d h$ locus, was sufficient to reveal the acquisition of a new strain in 7 cases, sequencing of more locuses revealed only two additional cases of a new strain acquisition. Then, in 8 out of 13 cases $(69.2 \%)$ of repeated recovery of $H$. influenzae at different timepoints, the new isolates represented, in truth, newly acquired strains. This is rather different from the observation of Sethi et al. ${ }^{1}$, who reported 145 cases of new $H$. influenzae acquisition versus 1503 cases of no new strain acquisition (8.7\%). Incorrect identification of $H$. haemolyticus as $H$. influenzae cannot be the cause of this difference, because we used the advanced version of database for MALDI-TOF MS identification ${ }^{10}$. However, in the study of Sethi et al. ${ }^{1}$, COPD patients were requested to come for screening for bacterial presence in sputum at regular monthly intervals regardless of their health status, unlike in our study, where sputum was sampled from patients who sought medical care or were treated during hospital stay. Then, the $8.7 \%$ frequency of new $H$. influenzae strain acquisition reported by Sethi et al. ${ }^{1}$ should represent the overall frequency of this event in COPD population, whereas the $61.5 \%$ frequency observed in our study should represent new strain acquisition in patients who typically sought medical care because of exacerbation. Such difference is not surprising in light of the 4.09 relative risk of exacerbation associated with $H$. influenzae new strain acquisition as reported by Murphy et al. ${ }^{3}$. To conclude, our data clearly demonstrate that the recovery of $H$. influenzae at the time of acute exacerbation should not be underestimated in chronic lung disease even in cases with a history of previous recovery of the same pathogen during stable disease because many 


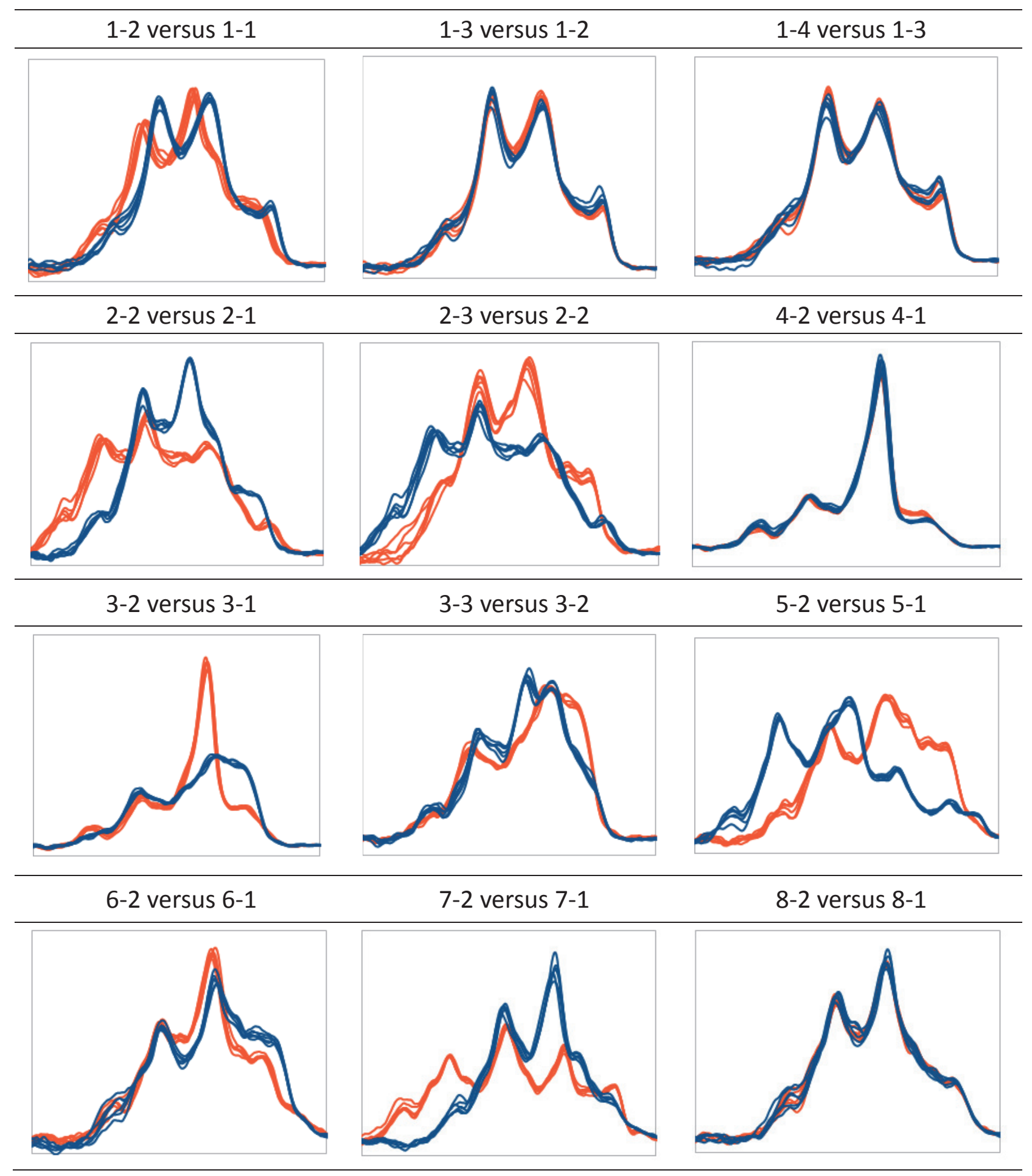

Fig. 3. Results of McRAPD typing represented by complex melting peak curves. Each plot represents a comparison of six replicates from one isolate versus six replicates from the other isolate, both from the same patient, e.g. 1-2 versus 1-1 plot represents comparison of melting curves obtained with the isolate No. 2 from patient No. 1 versus isolate No. 1 from the same patient. Replicates obtained with the same isolate are coded by the same colour. X axis: temperature points from 77.0 to $87.8^{\circ} \mathrm{C}$. Y axis: relative fluorescence intensity. Because of space limitations, twelve pairwise comparisons are demonstrated instead of all thirteen ones.

or even most of such cases do represent, in truth, acquisition of a new strain.

\section{MALDI-TOF MS strain typing}

MALDI-TOF MS protein profiling of whole bacterial cells has recently been introduced in most diagnostic labo- ratories as a routine technique for rapid and reliable species identification of bacteria. However, it also offers the opportunity to use unique strain-specific protein profiles for typing purposes. Application of MALDI-based typing has been reported for different species with variable success $\left(\right.$ ref. $\left.{ }^{11-17}\right)$. In this study, results of hierarchical cluster 
analysis of pairwise comparisons, visualized in the form of a dendrogram (Fig. 1), always indicated a clearly separate identity in each isolate, i.e. the acquisition of a new strain. However, in 5 cases, this was not in accordance with the results of MLST as a gold standard of strain typing. A complete dendrogram based on merged data from triplicates (Fig. 2) suggested identity of isolates 1-3 and 1-4 that belong to the same MLST type 124, unfortunately, the isolate 1-2 that also belongs to the MLST type 124 clustered separately. Moreover, the complete dendrogram suggested identity of isolates that were found unrelated by MLST analysis, and, on the other hand, clearly separated isolates of the same strain as established by MLST. For more detailed insight, we inspected the spectra of isolates $1-2,1-3,1-4$ and 7-1 visually. All of these isolates clustered more or less together in a complete dendrogram (Fig. 2), but we were not able to identify any robust strain-specific signals shared by 1-2, 1-3 and 1-4 isolates. There were many minor peaks of the same molecular weight but different intensity shared by all of the isolates. The clustering observed in the complete dendrogram is then mainly based on matching large numbers of low-intensity peaks that are rather variable in different isolates. Possibly, protein profiles are not stable enough in $H$. influenzae when isolates are recovered at different timepoints from the host organism. This conclusion is also supported by our observation of mixed clustering of two isolates recovered from pharyngeal swab and nasal swab, which correctly indicated the same strain identity when samples were taken from different locations of the same patient at the same timepoint (unpublished data). In addition, processing of extensive numbers of isolates in one run is not practicable because of the labour intensive character of the technique. Then, smaller groups of isolates need to be processed at different timepoints and instrumental stability cannot be always fully warranted. Taken together, MALDI-TOF MS was unable to discriminate new $H$. influenzae strains acquisition at the settings used in our study. Its performance might be improved by careful optimization, and it still may be useful for the discrimination of true outbreaks from accidental accumulation of sporadic cases, on the condition the sampling and processing is performed in a rather limited time frame. Also, improved techniques of MSPs' clustering may be able to provide more accurate results, as demonstrated with the dynamic cutoff method in a study of Achromobacter xylosoxidans epidemic strain among Belgian CF patients ${ }^{17}$.

\section{McRAPD strain typing}

Although RAPD has been widely used in microbial typing because of its easy and economic performance, including for $H$. influenzae ${ }^{18}$, it has also been criticized for poor reproducibility. Since we were aware of this drawback, including factors that influence the outcome of RAPD ${ }^{18}$, we strove to prevent this problem in several ways. First, we used a DNA extraction kit that proved to provide stable yields of high quality DNA in bacteria. Second, we prolonged the time from the annealing to the extension step to $7 \mathrm{~min}$, which should result in

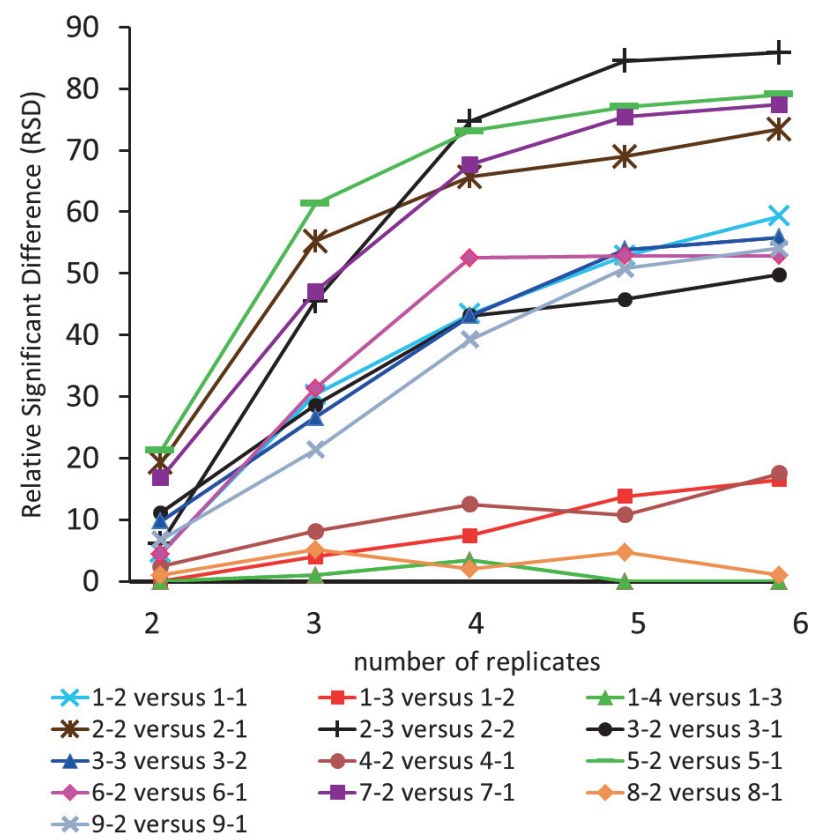

Fig. 4. Relative significant difference (RSD) values calculated in silico, to model the situation when less than six replicates per isolate would be included. Each curve represents one pairwise comparison of McRAPD data acquired with two isolates, e.g. 1-2 versus 1-1 curve represents comparison of the isolate No. 2 from patient No. 1 versus isolate No. 1 from the same patient. RSD values increase with increasing number of replicates included into calculation. Starting from three replicates, difference between the group of isolates belonging to the same strain (RSD $<20 \%$ ) and the group of isolates representing different strains (RSD $>20 \%$ ) is clearly visible.

more stable patterns ${ }^{9}$. Third, we performed each reaction in 6 replicates to be able to distinguish which part of RAPD pattern variability was due to the technique itself and which truly represented interstrain variation. Fifth, we used high resolution melting analysis to obtain hard numerical data and thus avoid possible bias when deciding which bands are too faint to be included into comparison of banding patterns, as it can happen in conventional RAPD. Sixth, we used a commercial PCR master mix that includes hot-start polymerase and third generation intercalating fluorescent dye, which proved to perform excellently acc. to our previous experience. Melting analysis of RAPD, termed McRAPD, was first used by our group for purposes of yeast species identification ${ }^{19}$, but later proved to be useful for strain typing in several other settings ${ }^{17,20-22}$. In this study, McRAPD provided excellent results that were in full accordance with the results of MLST, which is considered the gold standard for microbial typing. Identity or difference of melting patterns was easy to evaluate even by simple visual inspection of plots if samples were run in replicates, as demonstrated in Fig. 3. Numerical processing clearly confirmed this impression. Moreover, no more than three replicates were needed for easy and clear cut differentiation between Relative Significant Difference 
(RDS) values coming from pairs of isolates of the identical strain and those coming from pairs belonging to different strains (Fig. 4). In another independent study that directly compared McRAPD to MALDI-TOF MS typing in Achromobacter xylosoxidans recently, McRAPD also proved to be non-inferior ${ }^{17}$.

However, we should be aware of possible limitations of this promising technique. In this study, the principal aim of which was to evaluate differences between limited numbers of pairs of isolates, McRAPD worked excellently. If large numbers of isolates are studied with many resulting McRAPD patterns, the probability that profiles of two unrelated strains will appear related will increase. Of course, McRAPD performance will also vary with the different primers used in different species, as it is the case of any typing technique except for techniques that rely on extensive sequencing (MLST). Also, McRAPD results will always vary with different amplification platforms and dye chemistries, and even inter-run reproducibility may still pose a challenge. This effectively hinders inter-laboratory reproducibility and comparison of data. However, its easy, rapid and economic performance with the potential of automated processing of numerical data makes McRAPD a suitable candidate for routine discrimination of new strain acquisition, as described in this study in $H$. influenzae.

Acknowledgment: This work was supported by the Ministry of Health of the Czech Republic (NT/13560), by RVO (61989592), by IGA LF UP (IGA_LF_2016_022), and by the Ministry of Education, Youth and Sports of the Czech Republic (LO1304).

Author contributions: VR: project leader, collection of isolates, McRAPD typing and data processing, manuscript preparation; NS: MALDI-TOF MS typing; DS: sequencing; JB: MALDI-TOF MS species identification; JZ, MZ, and PJ: selection of patients, acquisition of informed consent, sampling; LN: conventional strain typing and PCR typing; RN: DNA extraction, isolate collection maintenance, data management.

Conflict of interest statement: The authors state that there are no conflicts of interest regarding the publication of this article.

\section{REFERENCES}

1. Sethi S, Evans N, Grant BJB, Murphy TF. New strains of bacteria and exacerbations of chronic obstructive pulmonary disease. N Engl J Med 2002;347:465-71. doi:10.1056/NEJMoa012561.

2. Sethi S, Wrona C, Grant BJB, Murphy TF. Strain-specific Immune Response to Haemophilus influenzae in Chronic Obstructive Pulmonary Disease. Am J Respir Crit Care Med 2004;169:448-53. doi:10.1164/rccm.200308-11810C.

3. Murphy TF, Brauer AL, Sethi S, Kilian M, Cai X, Lesse AJ. Haemophilus haemolyticus: A Human Respiratory Tract Commensal to Be Distinguished from Haemophilus influenzae. J Infect Dis 2007;195:81-9. doi:10.1086/509824.

4. Finney LJ, Ritchie A, Pollard E, Johnston SL, Mallia P. Lower airway colonization and inflammatory response in COPD: a focus on Haemophilus influenzae. Int J Chron Obstruct Pulmon Dis 2014;9:1119-32. doi:10.2147/COPD.S54477.

5. Plachy R, Hamal P, Raclavsky V. McRAPD as a new approach to rapid and accurate identification of pathogenic yeasts. J Microbiol
Methods 2005;60:107-13. doi:S0167-7012(04)00251-9 [pii] r10.1016/j.mimet.2004.09.003 [doi].

6. Meats E, Feil EJ, Stringer S, Cody AJ, Goldstein R, Kroll JS, Popovic T, Spratt BG. Characterization of Encapsulated and Noncapsulated Haemophilus influenzae and Determination of Phylogenetic Relationships by Multilocus Sequence Typing. J Clin Microbiol 2003;41:1623-36. doi:10.1128/JCM.41.4.1623-1636.2003.

7. Jolley KA, Maiden MCJ. BIGSdb: Scalable analysis of bacterial genome variation at the population level. BMC Bioinformatics 2010;11:595. doi:10.1186/1471-2105-11-595.

8. Mori E, Lio P, Daly S, Damiani G, Perito B, Fani R. Molecular nature of RAPD markers from Haemophilus influenzae Rd genome. Res Microbiol 1999;150:83-93.

9. Benter T, Papadopoulos S, Pape M, Manns M, Poliwoda H. Optimization and reproducibility of random amplified polymorphic DNA in human. Anal Biochem 1995;230:92-100.

10. Zhu B, Xiao D, Zhang H, Zhang Y, Gao Y, Xu L, Lv J, Wang Y, Zhang J, Shao Z. MALDI-TOF MS distinctly differentiates nontypable Haemophilus influenzae from Haemophilus haemolyticus. PLoS One 2013;8:e56139.

11. Jadhav S, Gulati V, Fox EM, Karpe A, Beale DJ, Sevior D, Bhave M, Palombo EA. Rapid identification and source-tracking of Listeria monocytogenes using MALDI-TOF mass spectrometry. Int J Food Microbiol 2015;202:1-9. doi:10.1016/j.ijfoodmicro.2015.01.023.

12. Khennouchi $\mathrm{NC}$ el $\mathrm{H}$, Loucif $L$, Boutefnouchet $N$, Allag $H$, Rolain J-M. MALDI-TOF MS as a Tool To Detect a Nosocomial Outbreak of Extended-Spectrum- $\beta$-Lactamase- and ArmA MethyltransferaseProducing Enterobacter cloacae Clinical Isolates in Algeria. Antimicrob Agents Chemother 2015;59:6477-83. doi:10.1128/ AAC.00615-15.

13. Carannante A, De Carolis E, Vacca P, Vella A, Vocale C, De Francesco MA, Cusini M, Del Re S, Dal Conte I, Cristaudo A, Ober P, Sanguinetti $M$, Stefanelli P. Evaluation of matrix-assisted laser desorption ionization-time of flight mass spectrometry (MALDI-TOF MS) for identification and clustering of Neisseria gonorrhoeae. BMC Microbiol 2015;15:142. doi:10.1186/s12866-015-0480-y.

14. Spinali S, van Belkum A, Goering RV, Girard V, Welker M, Van Nuenen M, Pincus DH, Arsac M, Durand G. Microbial typing by matrix-assisted laser desorption ionization-time of flight mass spectrometry: do we need guidance for data interpretation? J Clin Microbiol 2015;53:7605. doi:10.1128/JCM.01635-14.

15. Rim JH, Lee $Y$, Hong SK, Park Y, Kim M, D'Souza R, Park ES, Yong $D$, Lee K. Insufficient Discriminatory Power of Matrix-Assisted Laser Desorption Ionization Time-of-Flight Mass Spectrometry Dendrograms to Determine the Clonality of Multi-Drug-Resistant Acinetobacter baumannii Isolates from an Intensive Care Unit. Biomed Res Int 2015;2015:535027. doi:10.1155/2015/535027.

16. Koláčková I, Štromerová N, Bardoň J, Pudová V, Karpíšková R. Potential use of mass spectrometry for subtyping of Campylobacter. Klin Mikrobiol a Infekcní Lékarství 2015;21:68-73.

17. Cools $P, H o$ E, Vranckx K, Schelstraete P, Wurth B, Franckx H, leven G, Van Simaey L, Van Daele S, Verhulst S, De Baets F, Vaneechoutte M. Epidemic Achromobacter xylosoxidans strain among Belgian cystic fibrosis patients and review of literature. BMC Microbiol 2016;16:122. doi:10.1186/s12866-016-0736-1.

18. Dhooge I, Vaneechoutte M, Claeys G, Verschraegen G, Van Cauwenberge P. Turnover of Haemophilus influenzae isolates in otitis-prone children. Int J Pediatr Otorhinolaryngol 2000;54:7-12.

19. Trtkova J, Pavlicek P, Ruskova L, Hamal P, Koukalova D, Raclavsky V. Performance of optimized McRAPD in identification of 9 yeast species frequently isolated from patient samples: potential for automation. BMC Microbiol 2009;9:234.

20. Tulsiani SM, Craig SB, Graham GC, Cobbold RC, Dohnt MF, Burns M-A, Leung LK, Field HE, Smythe LD. High-resolution melt-curve analysis of random-amplified-polymorphic-DNA markers, for the characterisation of pathogenic Leptospira. Ann Trop Med Parasitol 2010;104:151-61. doi:10.1179/136485910X12607012374037.

21. Deschaght P, Van Simaey L, Decat E, Van Mechelen E, Brisse S, Vaneechoutte M. Rapid genotyping of Achromobacter xylosoxidans, Acinetobacter baumannii, Klebsiella pneumoniae, Pseudomonas aeruginosa and Stenotrophomonas maltophilia isolates using melting curve analysis of RAPD-generated DNA fragments (McRAPD). Res Microbiol 2011;162:386-92.

22. Hamal P, Hanzen J, Horn F, Trtkova J, Ruskova L, Vecerova R, Ruzicka F, Vollekova A, Raclavsky V. Usefulness of McRAPD for typing and 
importance of biofilm production in a case of nosocomial ventriculoperitoneal shunt infection caused by Candida lusitaniae. Folia Microbiol (Praha) 2011;56:407-14.
Supplemental Material:

The online version of this article (doi: 10.5507/ bp.2016.050) offers supplementary material. 